\title{
The Pharmacy Only Visit Care Model (POV) and its Effects on the Quality of Care of HIV Patients in Uganda. A Case Study of Mulago National Referral Hospital HIV Clinic. Clients' Perspective
}

\author{
J.C. Okiria ${ }^{1}$ H. Wasswa ${ }^{2}$ \\ ${ }^{1,2}$ International Health Sciences University-Kampala Uganda
}

\begin{abstract}
This study aimed at evaluating the POV care model and its effects on the quality of care of HIV patients in Mulago HIV clinic. Its specific objectives were to; determine the effect of the pharmacy only visit care model on the level of patient satisfaction in Mulago HIV clinic; assess the effects of the pharmacy only visit care model on Adherence of patients; and assess the benefits of the pharmacy only visit care model to patients on this model in Mulago HIV clinic. Overall the clients were satisfied with the POV model and the level of patients' adherence to treatment has greatly improved. The Model has also benefited the clients in terms of time, cost saved in seeking care. Increased autonomy, confidence, self management, and trust from doctors have been realized by the clients under the POV.
\end{abstract}

Keywords: Pharmacy only visit, Quality of care, Level of patient satisfaction, Adherence, Waiting time, Antiretroviral therapy.

\section{Introduction}

The Pharmacy Only Visit (POV) model is an innovative intervention that was developed to address the challenge of high patient numbers escalating in HIV/AIDS clinics specifically located in resource limited settings. It was enrolled within Mulago HIV clinic, one of the biggest HIV clinics run by Makerere University Joint AIDS program (MJAP), a PEPFAR funded program offering HIV care and treatment to over $62,000 \mathrm{HIV}$ infected patients. The model was rolled out in 2009 (Maselle E. et al., 2012)

In this model, patients who are stable with ART adherence levels $\geq 95 \%$ and a Karnofsky score $>90$, are assessed by the clinician or doctor once every 4 months, but visit the pharmacy every two months for ART refills for patients on ART; and for Non ART patients, reviewed every after 6 months but visit the pharmacy every after 3 months to reduce clinician visits thus clinic waiting time. At the time of the study $20 \%$ of patients on care were on POVs.

This study addressed the question as to whether the POV care model improves patient quality of care or not in the patients perspective.

\section{Methods}

\section{Study Setting}

Mulago HIV clinic is a specialized HIV treatment center within Mulago national referral hospital. Mulago Hospital is found in Kawempe division which is one of the five administrative divisions of Kampala city. It approximately covers an area of $30 \mathrm{~km}^{2}$ with composition of 18 parishes, each made up of 3 to 7 villages. Mulago HIV clinic is the referral HIV treatment center for Kawempe division, with an estimated daily attendance of 450 HIV patients. 12,400 of patients on care are on the POV care model.

\section{Study Design}

A cross-sectional study was conducted, using a mixed methods research approach, where both qualitative and quantitative data was collected (Onwuegbuzie \& Johnson, 2006; Greene, 2006; Greene, Caracelli, and Graham, 1989).employing both quantitative and qualitative methods.

\section{Target Population}

The target population consisted of HIV patients on the POV model in all clinics operated by MJAP

\section{Study Population}

The study population consisted of HIV patients on the POV model in Mulago HIV Clinic between 2009 and 2015

\section{Unit of analysis}

The unit of analysis was a HIV patient on the POV care model of treatment

\section{Sample Size}

The sample size of the number of patients who participated in the study regarding the effect of the POV care model on the Quality of care of HIV patients was calculated using the Yamane (1967) formula, and was 388 patients

\section{Data collection}

\section{Quantitative Data}

Quantitative data was collected using a pre-tested, semistructured questionnaire administered by the principal investigator and trained research assistants to the selected respondents. A retrospective data check was conducted on data of the respondents from 2009 to 2015.

\section{Qualitative Data}

Focus group discussions consisting of 8-12 participants on the POV care model were conducted by the principal investigator and trained researcher assistants. Three FGDs 


\section{International Journal of Science and Research (IJSR) \\ ISSN (Online): 2319-7064 \\ Index Copernicus Value (2013): 6.14 | Impact Factor (2015): 6.391}

were conducted, for each group there was a modulator to guide the discussions, a secretary and the principal investigator to record the proceedings. The FGDs were conducted in Luganda and English the language commonly used.

\section{Data Analysis}

\section{Quantitative data analysis}

Data was entered in data editor and analyzed using STATA statistical program version 12. Descriptive analysis was employed for the basic clinic characteristics, level of patient satisfaction and adherence to treatment

\section{Qualitative data analysis}

This was performed using theme and content qualitative methods. All transcripts were read several times to identify themes and categories. After discussion a coding frame was developed and transcripts coded by the author. This process was used to develop categories, which were then conceptualized into broad themes after further discussion. Representative quotes were selected to illustrate identified themes.

\section{Results}

\section{Quantitative results}

Results from the study showed an overall satisfaction with POV care services and support. A majority of POV patients $381(98.20 \%)$ reported complete satisfaction. Furthermore, the findings showed that with introduction of the POV care model, the waiting time at the pharmacy reduced to less than 15 minutes with $91.49 \%$ of the POV patients giving an utmost average of 10 minutes per wait. Results from the study also found out under the dimension of humanity of care; A majority of respondents, $362(93.30 \%)$ agreed that they were completely satisfied with the attitude of the POV attending staff towards serving them. Satisfaction with respect for privacy at the pharmacy was significantly high (94.85\%). However, 255(66.49\%) POVs had knowledge of the suggestion box and out of the 255 only 7 had ever used it. A total of $323(83.25 \%)$ of the respondents agreed that first come first serve criteria was being followed at the pharmacy. Results on adherence to treatment showed that a majority of POV patients $316(84.44 \%)$ picked their medication in the recommended clinic official time of $7 \mathrm{am}$ to $12 \mathrm{pm}$ in the morning. $83(21.39 \%)$ POV patients had ever missed taking their medication in the last 4 months. A majority 305 (78.61\%) had never missed taking their medication. Findings of this study also show that an overwhelming majority of the respondents (97.94\%) love the POV care model. Only $2.06 \%$ said they were not sure. However the study also found out that there are a number of issues POV patients were dissatisfied with.

\section{Qualitative results}

The majority number 380 (97.94\%), expressed a number of things they like most about the POV care model and vowed to keep meeting the necessary POV requirements like adherence to medication, keeping clinic appointments and time, and reporting any acquired conditions to doctors in order to be kept on this model. These reasons were grouped into themes of Time, Cost, Health, Autonomy and self management, Adherence, Convenience and Confidence and trust from doctors as expounded below:

\section{Time}

The theme of time was further sub categorized into 3 subthemes, namely; waiting time, time and work, and time and family;

\section{Waiting Time}

"POVs have a small waiting time at the pharmacy"

A number of patients reported that one of the main reasons they love being on the POV care model was because they do not have to wait so long at the pharmacy for drugs any more. This supports the earlier quantitative findings that found out that the mean waiting time at the pharmacy during the POV appointment is less than 10 minutes

\section{Time and work}

"POVs help me settle on my Job without worries of being absent because of frequent clinic visits"

This can be attributed to the fact that when a patient is on the POV care model they ideally see their doctor every after 4 or 6 months. For working patients, this enables them to settle at their jobs without any interruptions of frequent clinic visits to see their doctor.

"I have enough time to do my business"

A number of patients who are operating their own businesses were pleased at the amount of time the POV care model creates for them out of the clinic. With this created time, they are able to uninterruptedly operate their own businesses successfully.

\section{Time and family}

"POVs enable me spend more time with my family"

Patients with families especially the mothers professed with delight that being put on the POV care model treatment plan gave them enough time to settle at home to ably and satisfactorily look after their husbands and young children. They stressed that they practically only have to visit the clinic every after 4 or 6 months, because the POV appointment in between, doesn't take them any significant time in the clinic.

\section{Cost}

"POVs are cost effective. We no longer spend a lot of money on transport because visits are done every after 2 or 3 months unlike the past where you were required to see the doctor often."

Before introduction of the POV care model in the clinic, patients practically visited the clinic every month for their doctor review. It must appreciated that a number of patients in the Mulago HIV clinic reside upcountry or out of Kampala and transportation cost for these long journey commutations are so expensive to sustain. For example one of the respondents revealed that they need to save approximately 60,000 shillings(18 USD) for transport to and fro, to commute from Mubende to Mulago HIV clinic. Such high transportation costs would be unsustainable if clinic visits were still being conducted monthly.

\section{Health \\ "We no longer fall sick often"}

One of the main POV topics in the education sessions given in the clinic to the patients is the significance of good adherence while on the POV medication. Therefore patient's health stability while on the POV care model can be 


\section{International Journal of Science and Research (IJSR) \\ ISSN (Online): 2319-7064 \\ Index Copernicus Value (2013): 6.14 | Impact Factor (2015): 6.391}

attributed to the improved adherence to their medication. This can be further supported by earlier quantitative findings about the effect of the POV care model on adherence, that reveal very good adherence levels to medication by the biggest proportion of patients on the POV care model, 305 $(78.61 \%)$

"There is no longer any need for me to come to the hospital frequently."

A number of patients revealed that because they generally feel their health status is good, they don't have any ailments or disturbing health conditions any more, there is no longer any need for them to come to the hospital frequently, and hence they felt that the POV care model greatly suited them and that they were pleased.

\section{Autonomy and Self Management \\ "The clinic puts trust in me to manage my own medication" A majority of the patients on the POV care model expressed their delight at the level of trust the clinic has bestowed them by allotting them medication quantities of 2 or 3 months, and also giving them permission to monitor their own health out of the clinic for a long duration of time. However, patients on the POV care model are advised to return to the clinic and see a doctor in case they have any health problems that have emerged up during their stay out of the clinic.}

"Being on POVs enables me negotiate on a convenient POV appointment date with my doctor."

Self reports POV patients showed that a number of POV patients enjoyed the negotiating powers that have been granted to them by their doctors on issues pertaining allocation of the POV appointment date. A majority of patients disclosed that they were now able to negotiate on a convenient POV appointment date with their doctor by the virtue of being on the POV care model.

"POVs give me a sense of responsibility to make my schedules a head of time"

A number of patients on the POV care model revealed that now they are able to make plans for their treatment visits and taking of medication very well, given the sense of responsibility they feel is entrusted to them by the clinic. They confessed that this is the main reason why they do not miss their POV appointment dates.

\section{Adherence \\ "Being on POVs motivates me to keep taking my medication well" \\ Patients on the POV care model divulged that because of the so many associated benefits they are enjoying from the being on the model, such as less waiting time, the long time out of the clinic, trust entrusted to them by their doctors, and others, it motivates them to keep taking their medication very well because they do not want to be withdrawn from the POV treatment plan because of poor adherence and then consequently lose all the associated benefits.}

\section{Convenience}

"POVs enable you to come and pick your drugs ahead of time. In cases where you are going to travel away from home, you can come a day before."
Patients were so delighted with convenience that comes with being on the POV care model. A number of them cited out scenarios where they were having travel commitments far away from Kampala but came to the pharmacy before the POV appointment date and explained to the pharmacy staff and they were able to pick their drugs a head of time.

"I stay very far so the POV care model is convenient for me, I don't have to frequent the clinic"

A numbers of patients were delighted to the fact that they don't have to frequent the clinic any more, and all this was attributed to convenience the POV care model brings.

"There is no congestion when you come for POVs" A majority of the respondents expressed their joy at experiencing no congestions at the pharmacy when they come to pick their POV medication. This can be attributed to the fact that POV appointments are allocated morning hours between $7 \mathrm{am}$ to $12 \mathrm{pm}$. These are the less busy hours of the clinic, so patients on the POV care model are specifically given all the attention they need by the pharmacy staff.

"I enjoy the duration out of the clinic. It is long and comfortable."

A majority of respondents expressed their delight at the long duration they enjoy out of the clinic. They reported that it was long and comfortable

"When you come early for you POV, you go away early." Serving hours for POV appointments are very convenient for POV patients who come early for their medication are able to be worked on very fast and then leave the clinic fast

\section{Confidence from doctors}

"My doctors show confidence in me"

Self reports from a majority of patients on the POV care model revealed that they were pleased with the confidence doctors are showing them in matters regarding management of their medication and treatment plan

\section{Discussion}

In this paper we address a critically important aspect of ART-scale up in resource limited setting through introduction of the POV care model and assessing its effect on the quality of care of HIV patients with a case study of Mulago HIV clinic.

Level of patients' satisfaction on the POV care model in Mulago HIV clinic. Understanding patients' perspectives is a vital step in the efforts to improve the quality of the POV care and healthcare services as a whole in Mulago HIV clinic (WHO Report, 2009).

The findings show that with introduction of the POV care model, the waiting time at the pharmacy on the POV appointment is less than 15 minutes. In fact $91.49 \%$ of the POV patients gave an utmost average of 10 minutes per wait, and reported that they were fully satisfied with the Model. Other studies conducted in sub-Saharan Africa too identified lengthy waiting times as one of the biggest deterrence to patient satisfaction and retention (Colebunders R. et al., 2007) 


\section{International Journal of Science and Research (IJSR) \\ ISSN (Online): 2319-7064 \\ Index Copernicus Value (2013): 6.14 | Impact Factor (2015): 6.391}

Furthermore, findings of this study show that most of the POV patients $(97.42 \%)$ are fully satisfied with the timely availability of pharmacy staff to serve them POV medication. This is in line with a time motion study done in Uganda by Wanyenze et al., (2010), where they cited out that availability of health staff in HIV clinics is not the main problem, but rather the organization of services which results in inefficiencies and delays.

The study also found out that the greatest proportion of patients $(93.56 \%)$ on the POV care model were completely satisfied with the availability of all drugs at Mulago HIV clinic pharmacy. Unlike similar studies done about patient satisfaction in public health facilities where lack of availability of drugs is a major cause of patient dissatisfaction,(Castelnuovo B. et al., 2015). However, the small proportion of POV patients confessed dissatisfaction with the issue of availability of pharmacy staff, provides an incite of the areas to improve in relation to organizations of the staff.

In terms of the dispensing practices at the Mulago HIV clinic pharmacy, the findings of the study show that a majority of POV patients are completely satisfied with the clear and understandable instructions about POV medications and services given to them by the pharmacy (98.71\%). A report made by the Ministry of Health on the study of client satisfaction with health services in Uganda revealed that patient satisfaction was high with the level and skills of healthcare personnel (MOH Report, 2010), whch is in line with this study

Furthermore, findings of this study show high patient satisfaction with the Promptness of care given to them at the pharmacy waiting area 97.94\% (380); how their concerns are addressed 97.94\% (380); talks about POV services made in education sessions $81.19 \%$ (315); Pharmacy support in reminding them to keep their appointments during dispensing $80.67 \%$ (313); and the initiative taken by the clinic staff to inform them of the changes in POV services time to time $91.75 \%$ (356). This compares favorably with results from other earlier studies conducted by (Jenkinson C. et al., 2011).

Results from this study also found out under satisfaction with humanity of care, satisfaction with attitude of POV attending staff $(93.30 \%)$ was as high as satisfaction with Dignity and respect given by POV staff (93.81\%) and attention given by POV staff (93.81\%). This is in line with findings from a report by $\mathrm{MOH}{ }^{[5]}$ which reported high satisfaction among the above three indicators of satisfaction.

The findings further identified that satisfaction with respect to privacy at the pharmacy was significantly high (94.85\%) and highly associated with formal education, age and economic status. This still is in line with the $\mathrm{MOH}$ report (2010) ${ }^{[5]}$ for client satisfaction with Health services in Uganda.

The study found out an association between satisfaction and patient characteristics. Occupation (economic status), formal education and age were found to be important factors of satisfaction. This was still in line with studies conducted (Crane HM et al., 2007).

\section{Adherence to medication and POV appointment}

This section provides discussion of results from a number of indicators allocated to tracking patient's adherence towards medication and POV appointment while on the POV care model. These indicators were: Compliance to recommended official time for picking POV medication in the clinic; adherence to POV appointment; adherence to POV medication; and carrying of pill balances to the pharmacy when picking POV medication.

The findings show that the greatest proportion of the POV patients $84.44 \%$ ( 316 ) were compliant to the recommended official time of picking POV medication at the clinic of $7 \mathrm{am}$ to $12 \mathrm{pm}$ in the morning. However it must be noted that results showed a significant association between time of picking POV medication at the pharmacy and waiting time $(\mathrm{OR}=10.43,95 \% \mathrm{CI}(4.84-22.50), \mathrm{P}=0.000)$. This can be explained that patients that picked their medication in the morning hours had less waiting time at the pharmacy i.e. < 15 minutes, than patients who picked drugs in the afternoon (these had a longer waiting time at the pharmacy $>15$ minutes). This compares favorably to the study done by Wanyenze et al., (2010), on the evaluation of the efficiency of patient flow at 3 HIV clinics in Uganda. They found out that earlier hour visitation times improve the clinic flow consequently resulting into a reduction in waiting time. Furthermore the findings showed that the greatest proportion of POV patients $285(73.45 \%$ ) had never missed their POV appointment.

The study findings further showed that a majority of POV patients $78.61 \%$ are adherent to their medication. This is in line with the study done by (Ammassari et al., 2004).

Furthermore, the study found out significant associations between poor adherence and having too many pills at home $(\mathrm{P}=0.000)$, Forgetfulness $(\mathrm{P}=0.000)$, Being away from home $(\mathrm{P}=0.001)$, Being given a wrong appointment date $(\mathrm{P}=0.0001)$, Being given less pre-packed pills at the pharmacy $(\mathrm{P}=0.000)$. These finding are in line with findings from study conducted by Forgarty et al., (2007), on the factors associated to Adherence.

\section{Benefits of the Pharmacy only visit care model to the patient}

Findings of this study show that an overwhelming majority of the respondents $(97.94 \%)$ love the POV care model. This correlates with results from a study done by Castelnuovo et al., (2009), in an HIV clinic in Uganda. This study found out that there was reduction in the waiting time despite an increase in the number of patients after starting the nurse visits and pharmacy only visits.

Still under this section the study used a number of statements that related to the ideas about the POV care model to explore the perceived benefits enjoyed by patients on this model. The study revealed that approximately an average of $95 \%$ of the POV patients agreed that they were enjoying the following benefits: POVs are very convenient, decrease feelings of stress and tension for lining up in the 


\section{International Journal of Science and Research (IJSR) \\ ISSN (Online): 2319-7064 \\ Index Copernicus Value (2013): 6.14 | Impact Factor (2015): 6.391}

clinic, improve available time, give a sense of personal trust from the doctor, and give a sense of personal responsibility and autonomy.

\section{Recommendations}

Overall the POV model should be rolled out to all HIV clinics, however, the short falls in the POV service delivery can be resolved through implementation of the following recommendations.

\section{Level of patient satisfaction}

Although results from the study showed overall satisfactions with the POV care services and support. A proportion of patients expressed their dissatisfaction with some areas of the POV care model. This provides a critical platform for enhancing the POV care model to attain complete patient satisfaction, and hence a number of recommendations have been suggested:

1) Simplify the process or bureaucracy for POV patients to see a clinician in case one comes back with an opportunistic infection e.g. malaria, cough etc.

2) First come first serve criterion should be effectively implemented at the pharmacy and opening of pharmacy on time.

\section{Adherence to medication and POV appointment}

To improve adherence further of this category of patients, the following recommendations are suggested:

1) More effort to provide education sessions to patients on medication adherence and its importance, at all levels; counseling, clinician, nurse, peer educator, and pharmacy level.

2) Education sessions on proper storage practices of medication at home should be provided to POV patients since they take large quantities of medicines and stay out of the clinic for a long period of time, which might compromise the quality of drugs in their custody if proper storage conditions are not adhered to

\section{Benefits of the pharmacy only visit care model to patients}

More education sessions on the Pharmacy only visit model should be made.

The research recommends further research to;

1) Evaluation of clinical outcomes of HIV patients as a result of the POV care model

2) Assessment of the effect of the POV care model on the quality of life of HIV patients

\section{References}

[1] Ammassari A, Antinori A, Aloisi MS, et al., (2004). Depressive symptoms, neurocognitive impairement, and adherence to highly active antiretroviral therapy among HIV-Infected persons. Psychosomatics; 45:395-402 [PubMed]

[2] Bauman M, et al., (2005). Correlates of physical activity: why some people physically active and others not?: Lancet, 380 (9838) 258-271
[3] Castelnuovo B, Babiguma J, Lamorde M, Muwanga A, Kambugu A, Colebunders R (2009). Improvement of the patient flow in a large urban clinic with high HIV seroprevalence in Kampala, Uganda. Int J STD AIDS; 20(2):123 - 124. [PubMed]

[4] Castelnuovo B, Musaazi J, Musomba R, Kiragga AN, et al., (2015). Quantifying retension during preantiretroviral treatment in large urban clinic in Uganda. BMC Infect Dis; 15-252 [PubMed]

[5] Colebunders R ,Bukenya T, Pakkers N, et al., (2007). Assessment of the patients flow at the infectious disease institute out-patient clinic, Kampala, Uganda. AIDS Care; 19(2):149-151

[6] Crane HM, Lober W, Webster E, Harrington RD, Crane PK, et al., (2007). Routine collection of patient-reported outcomes in an HIV clinic setting: the first 100 patients. Cum. HIV Res 5:109-118

[7] Forgaty L, Roter D, Larson S, Burke J, Gillespie J, Levy R.(2007). Patient adherence to HIV medication regimen: A review of published and abstract reports. Patient Education and counseling; 46:93-108 [PubMed]

[8] Jenkinson C, Coulter A, Buster S, Richard N, Chandola T. (2011). Patient's experience and satisfaction with Health care: Results of a questionnaire study of specific aspects of care. Quality safety Health Care, Vol. 2011; 11-335-339 Journal of Technological Assessment in Health Care, 11, 443-455.

[9] Maselle E, et al., (2012), HIV-infected adults transitioned to Pharmacy Only Visits (POV) in an Urban HIV clinic in Uganda. International conference on AIDS and STIs in Africa. South Africa. 2012

[10] Ministry of Health (2010). The Health sector strategic plan III 2010/11-2014/15. Kampala: Ministry of Health Uganda.

[11] Wanyenze RK, Wagner G, Alamos S, et al., (2010). Evaluation of the efficiency of patient flow at three Ugandan HIV clinics AIDS patient care STDS; 24:441446 [PubMed]

[12] WHO Report (2009). Conquering suffering, enriching humanity. World Health Organization. Geneva 